\title{
Optimization of the Structural Parameters of Fisheye Contact Pairs by RSM
}

\author{
Maohua Xiao, Jun Guo, Xinhua Lu, Liping Shi, Jing Zhang, Hong Lin \\ College of Engineering, Nanjing Agricultural University, No.40, Dianjiangtai Road, Pukou Distinct, Nanjing 210031. \\ China. E-mail: xiaomaohua@njau.edu.cn,1010209791@qq.com,113905465@qq.com,125893610@qq.com, zhan- \\ gjing@njau.edu.cn,906869359@qq.com
}

\begin{abstract}
Fisheye contact pair is an important component of board level interconnection products wide range of applications. However, insertion and withdrawal force have great influence on the contact deformation due to their small size and complex structure. In this paper to the fisheye contact pairs of interconnection products as the research object, the main parameters were analyzed which could affect the contact deformation of fisheye structure, and factor analysis of them were performed. And three relatively more significant factors were extracted. The simulation based on Response Surface Methodology (RSM) is designed, And the experimental data was conducted regression fitting, getting the second regression model. The established quadratic regression model was analyzed based on MATLAB and the response of various factors on the insertion and withdrawal force, then the optimized parameter model of fisheye structure was obtained. The research shows that the quadratic regression model based on response surface methodology fitting accuracy was high and there were good practical value about it.
\end{abstract}

Keywords: Fisheye contact pair, Structural parameters, RSM, MATLAB, Design optimizing

\section{Introduction}

Currently, the board-level contact parts are widely used, and the contact failure also occurs frequently. According to NASA's statistics on the aeronautical loss rate, the failure caused by electronic components accounted for $40 \%$, of which $20 \%$ to $30 \%$ came from the failure of electrical connectors [1]. Structural parameters design of electrical connectors and plastic deformation caused by increasing wear of contact parts will result in contact failure [2][3][4], continuous mechanical vibration stress and frequent insertion will cause fretting wear of pins and jacks in electrical connectors [5], the corresponding electrical contact failure is one of the fundamental causes lead to electrical connector's function failure [6].

In the field of connector contact, experts have experimented on key factors, failure forms and reliability. Liang and his group have proposed a methodology to control the insertion force of brush electrical contact based on the depth of inserting [7]. Domestic and foreign scholars and manufacturers are committed to seeking high reliability, long-life electrical connectors [8]. And some scholars have proposed using numerical analysis to study contact reliability [9]. Therefore, it is necessary to test the reliability of interconnect products and optimize the design methodology. Most interconnect products in China rely on design experience [10][11][12], only dimensional chain calculation and electrical performance estimates. Repeated sample production, test validation and product improvement, will result in a great waste of cycles and resources [13].

Studies aiming at the influence of structure parameters on the fisheye contact pair's touching process and the change of the insertion force are less in the electrical connector [14]. To this end, this paper analyzes the dualfactor interaction effect bearing on fisheye contact and the influence of structural parameters on contact condition and effectiveness by response surface methodology. Firstly, use MATLAB to draw the relation curve surface of the insertion force and structure parameter [15][16][17], which reflecting the relationship between response and factor intuitively. Then find the second order model to fit it and study on the size of pulling force under different structural parameters. The formation of theoretical methodology and testing methodology can be applied to other areas, providing theoretical methodology and basis for practical design of fisheye contact parts.

\section{Optimizing structural parameters based on RSM}

In the application, the fish eyes enables the plate-level connector to be pressed into the Printed Circuit Bo$\operatorname{ard}(\mathrm{PCB})$, while its own structure will be deformed by extrusion, which produces a certain insertion and withdrawal force. Insertion and withdrawal force is the key mechanical property of fisheye contact piece, a main performance index in the connector assembling. Excessive pressure in the insertion process will cause assembly difficulty and affect insertion loss [12], so it is significant to study fisheye structure.

\subsection{Analysis of simulation factors}

In order to meet practical application conditions, the paper assumed that pressure is sufficiently large to minimize the insertion force. Because if only one parameter is adjusted to analyze the degree of influence, it is impossible to obtain the optimal solution. To find the optimal solution, this paper uses Placket-burman Experimental Design (PB) methodology to analyze how fisheye structure's various factors influence the insertion and withdrawal force. Through the manufacture experience and simulation results of the electric connectors, we found that the parameters which influence fisheye contact pair's insertion and withdrawal force mainly were fisheye structural parameters: the outer edge width, the root width, the inner hole length and the inner diameter width, as shown in Fig. 1. We chose two levels for each of the above four factors, named high and low levels, and values 
are shown in Tab. 1. Considering the problems this paper studying includes curved, a surface model is established with second order model to fit it, such as equation 1 .

$$
y=\beta_{0}+\sum_{i=1}^{k} \beta_{i} x_{i}+\sum_{i=1}^{k} \sum_{j=1}^{k} \beta_{i j} x_{i} x_{j}
$$

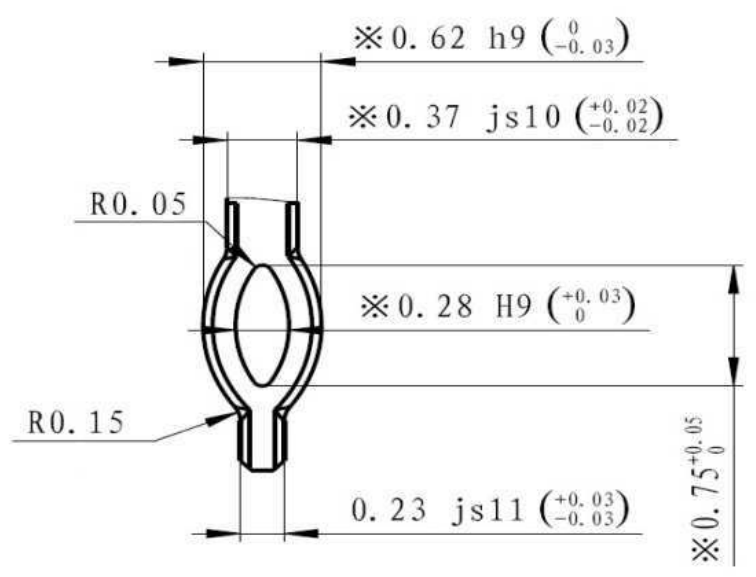

Fig. 1 Basic parameters of fisheye structure

\subsection{Establishment of insertion and withdrawal force model}

Using the multivariate regression equation to fit the data obtained by this experiment, a second-order response surface model is established based on the formula (1).

$$
\begin{aligned}
& F=\beta_{0}+\sum_{i=1}^{3} \beta_{i} x_{i}+\sum_{i=1}^{3} \sum_{j=1}^{3} \beta_{i j} x_{i} x_{j}=\beta_{0}+ \\
& \beta_{1} x_{1}+\beta_{2} x_{2}+\beta_{3} x_{3}+\beta_{12} x_{1} x_{2}+\beta_{13} x_{1} x_{3}+ \\
& \beta_{23} x_{2} x_{3}+\beta_{11} x_{1}{ }^{2}+\beta_{22} x_{2}{ }^{2}+\beta_{33} x_{3}{ }^{2}
\end{aligned}
$$

Where:

$F$ means the insertion or withdrawal force, $x_{1}, x_{2}$, $x_{3}$ respectively represent to the outer rim width, the root width, the inner diameter width, $\beta_{i j}$ is the coefficient of each variable. According to formula (2), we first used MATLAB to calculate the test data fitted by least square methodology, the fitting second order regression model of insert force are as formula 3 and 4 show.

$$
\begin{aligned}
F_{\text {insertion }} & =-114.5128+1123.4 x_{1}-951.0873 x_{2}-445.1348 x_{3} \\
& +4.1667 x_{1} x_{2}+3.4722 x_{1} x_{3}+145.8333 x_{2} x_{3} \\
& -776.9608 x_{1}{ }^{2}+1101.2 x_{2}{ }^{2}+600.6944 x_{3}{ }^{2} \\
F_{\text {withdrawal }} & =-99.8307+508.223 x_{1}-187.0539 x_{2}-154.1348 x_{3} \\
- & 154.1667 x_{1} x_{2}-170.1389 x_{1} x_{3}+429.1667 x_{2} x_{3} \\
- & 304.7386 x_{1}{ }^{2}+201.1765 x_{2}{ }^{2}+149.3056 x_{3}{ }^{2}
\end{aligned}
$$

The SPSS is used to fit data, the software excluded four dependent variables, and finally the fitting model is obtained as shown in equation 5 :

$$
\begin{aligned}
F_{\text {insertion }} & =-8.48+42.454 x_{1}-39.262 x_{2} \\
& +452.908 x_{2} x_{3}-131.828 x_{2}{ }^{2}-334.307 x_{3}^{2}
\end{aligned}
$$

Tab. 2 indicate that in the insertion force model, $\mathrm{R}=$ 0.9924 , interpretation rate $99.24 \%$, and the $\mathrm{P}$ value of the $F$ test is more than 0.0001 and less than 0.001 . It shows that the fitted force model is valid. However, in the withdrawal force model, $\mathrm{R}=0.8374$, the interpretation rate is not high, and the $\mathrm{P}$ value of the F test is 0.1298 , so the fit pull force model can't fit well. By using SPSS software to refit the pull-force model, the relevant information is proposed in Tab. 3, 4, 5 and 6.

Tab. 1 Fisheye structure factor level

\begin{tabular}{cccc}
\hline Variable & \multicolumn{3}{c}{ Horizontal value } \\
\hline Edge width $/ \mathrm{mm}$ & 0.56 & 0.62 & 0.68 \\
Inner diameter width $/ \mathrm{mm}$ & 0.22 & 0.28 & 0.34 \\
\hline
\end{tabular}

Tab. 2 Model evaluation parameters

\begin{tabular}{ccccc}
\hline Model & $\mathrm{R}$ & $\mathrm{F}$ & $\mathrm{P}$ & Error variance \\
\hline 1 & 0.9924 & 72.5444 & 0.0001 & 2.0056 \\
2 & 0.8374 & 2.8614 & 0.1298 & 2.3398 \\
\hline
\end{tabular}

Tab. 3 Abstract of withdrawal force model

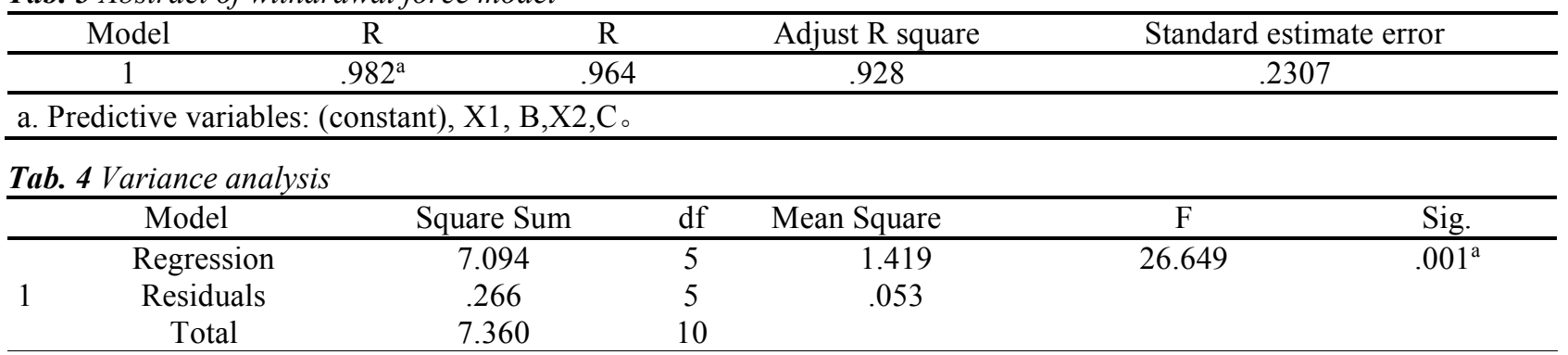

a. Predictive variables: (constant), F, X1, B, X2, C。

b. Dependent variables: Y 
Tab. 5 Regression Coefficients

\begin{tabular}{cccccc}
\hline \multirow{2}{*}{ Model } & \multicolumn{2}{c}{ Unstandardized Coefficients } & $\begin{array}{c}\text { Standardized Coeffi- } \\
\text { cients } \\
\text { Trial Version }\end{array}$ & t & Sig. \\
& $\mathrm{B}$ & Standard Error & & -.421 & .691 \\
(Constant) & -8.480 & 20.153 & .831 & 8.814 & .000 \\
$\mathrm{X}_{1}$ & 42.454 & 4.817 & -.915 & -.359 & .734 \\
$\mathrm{X}_{2}$ & -39.262 & 109.224 & -2.276 & -1.007 & .360 \\
B & -131.828 & 130.966 & -4.327 & -1.663 & .157 \\
C & -334.307 & 201.007 & 4.689 & 1.404 & .219 \\
F & 452.908 & 322.498 & & & \\
\hline
\end{tabular}

a. Dependent variables

By comparing Tab. 2 with Tab. 3, the $\mathrm{R}$ increased from 0.8374 to 0.964 is found, so the interpretation rate of the model shown in Model 2 was higher than that shown in equation 7 , which means that equation was more suitable. The F value in Tab. 4 is 26.649 and the corresponding $p$ value is over 0.001 but less than 0.05 , so the model could interpret the data, and the selected model was reasonable.

Finally, the insertion and withdrawal force and the fitting model of each factor was got as follows:

$$
\begin{gathered}
F_{\text {insertion }}=-8.48+42.454 x_{1}-39.262 x_{2} \\
\quad+452.908 x_{2} x_{3}-131.828 x_{2}{ }^{2}-334.307 x_{3}^{2} \\
F_{\text {insertion }}=-114.5128+1123.4 x_{1}-951.0873 x_{2} \\
-445.1348 x_{3}+4.1667 x_{1} x_{2}+3.4722 x_{1} x_{3} \\
+145.8333 x_{2} x_{3}-776.9608 x_{1}^{2}+1101.2 x_{2}{ }^{2} \\
+600.6944 x_{3}{ }^{2} \\
F_{\text {withdrawal }}=-8.48+42.454 x_{1}-39.262 x_{2} \\
\quad+452.908 x_{2} x_{3}-131.828 x_{2}{ }^{2}-334.307 x_{3}{ }^{2}
\end{gathered}
$$

\subsection{The response surface model of fisheye contact} pair

edge width and inner diameter width, the root width to $0.37 \mathrm{~mm}$ according to the practical experience is set, and set the response surface model, as shown in type 8,9 .

The relationship between the withdrawal force, outer

$$
\begin{aligned}
& F_{\text {withdrawal }}=-8.48+42.454 x_{1}-39.262 x_{2}+452.908 x_{2} x_{3}- \\
& 131.828 x_{2}^{2}-334.307 x_{3}^{2} \\
& F_{\text {insertion }}=-315.6608+1124.9417 x_{1}-391.1765 x_{3}+3.4722 x_{1} x_{3} \\
& -776.9608 x_{1}^{2}+600.6944 x_{3}^{2}
\end{aligned}
$$

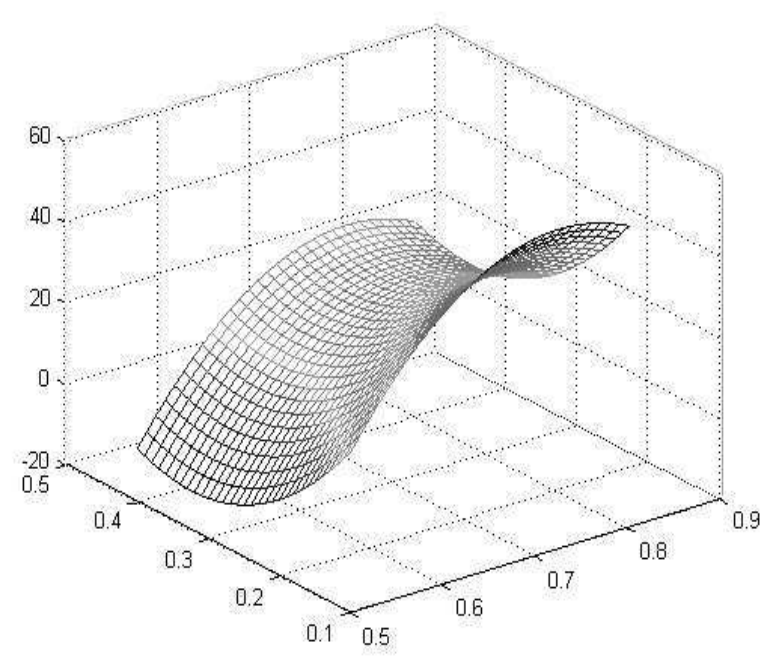

a) Insertion force surface diagram

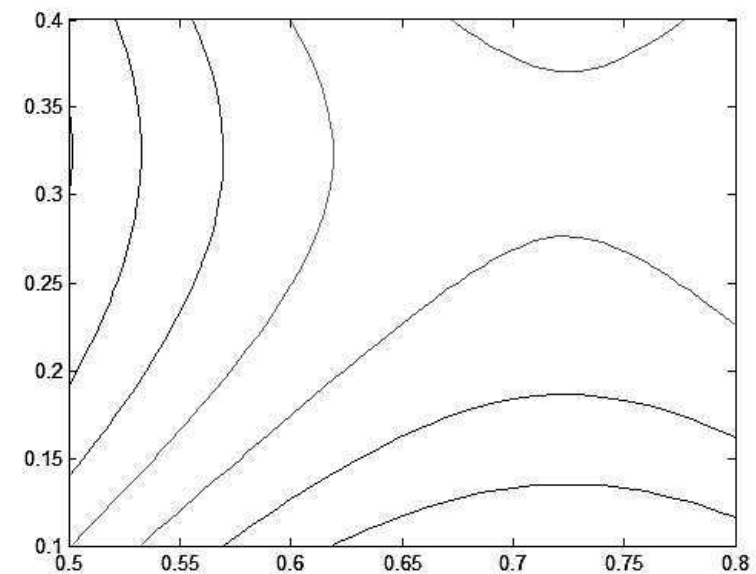

b) Insertion force contour map

Fig. 2 Insertion force surface diagram and contour map 


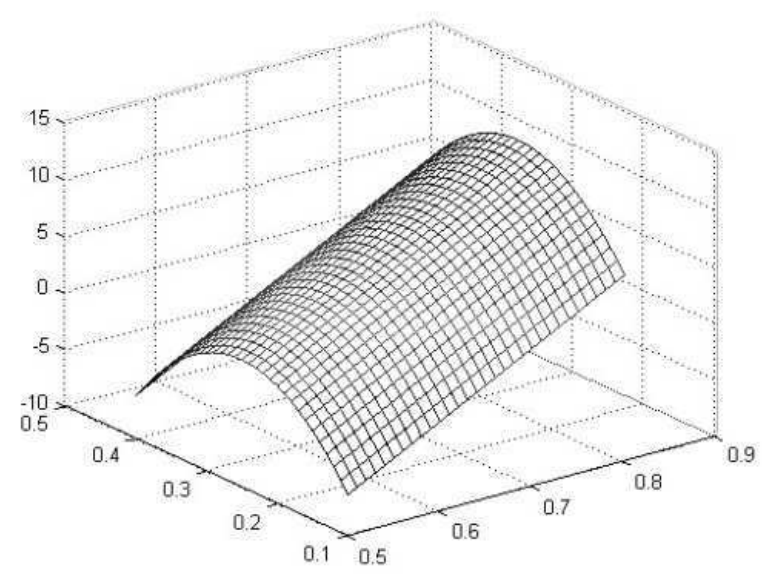

a) Insertion force surface diagram

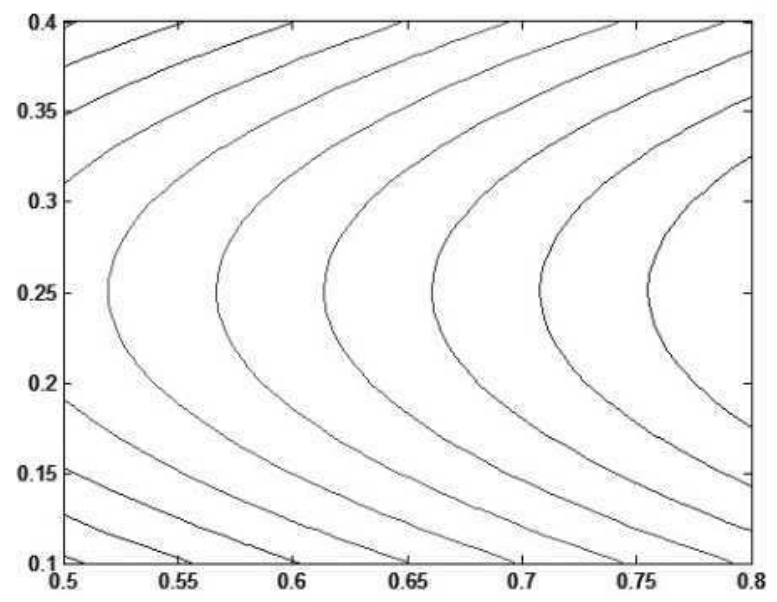

b) Insert force surface diagram

Fig. 3 Withdrawal force surface and contour map

The MATLAB is used to draw out the insertion and withdrawal force surface diagram, which indicated the interaction effect of the fisheye structure's outer edge and inner hole width on insertion and withdrawal force. It can be seen that the interaction between the two factors is clear from the graph. Under the circumstances that the root width remains unchanged, the smaller the outer edge width is, the smaller the insertion force is when the inner diameter is 0.32 [mm]. The withdrawal force increases with the width of the outer edge when the withdrawal force is near the width of $0.30[\mathrm{~mm}]$ in the inner diameter. From the contour map of the insertion force, we find that the larger the outer rim width, the larger the insertion force. The outer width chose 0.74 [mm] when insertion force is provided small enough, and the structural parameters in the case of the maximum ratio of insertion and withdrawal force are the optimized results. In summary, choosing edge width 0.74 [mm], inner hole width 0.32 [mm], the insertion force is the smallest, and withdrawal force is relatively large enough.

\section{Test and model comparison}

In order to prove the feasibility of optimizing structural parameters by the response surface methodology, the
15 pairs of two-factor interaction tests are set up by using fisheye contact parts as the test objects, with the outer edge width and the inner diameter width as factors control the inner diameter length and the root width unchanged.

This time, the plate-level interconnection structure was assembled to measure the insertion and withdrawal force between the fisheye structure and the printed plate plating hole by a pressure test machine. Servo system automatic insertion and withdrawal test machine is model $1220 \mathrm{HS}$. After the experiments, the computer would continuously change the amount of insertion and insertion and withdrawal force, and finally display the data on the scene.

The main technical parameters of the insertion and withdrawal force tester are as Tab. 6. This analysis accuracy can reach $0.01[\mathrm{~N}]$, the minimum fine-tuning distance is 0.01 [mm], the test speed range is $1-200$ $[\mathrm{mm} / \mathrm{min}]$, which can meet the fisheye contact pairs' insertion requirements. To make the experimental results more accurate, the 5 contact pieces with same structural parameters for each group are set, using the Model 1220HS testing machine, as shown in Fig. 4 and then we measured the peak of insertion and withdrawal force in each contact parts group, in order to calculated the $u$ ratio of withdrawal force to insertion force indirectly. The interpolation force and value analysis diagram are plotted as shown in Fig. 5.

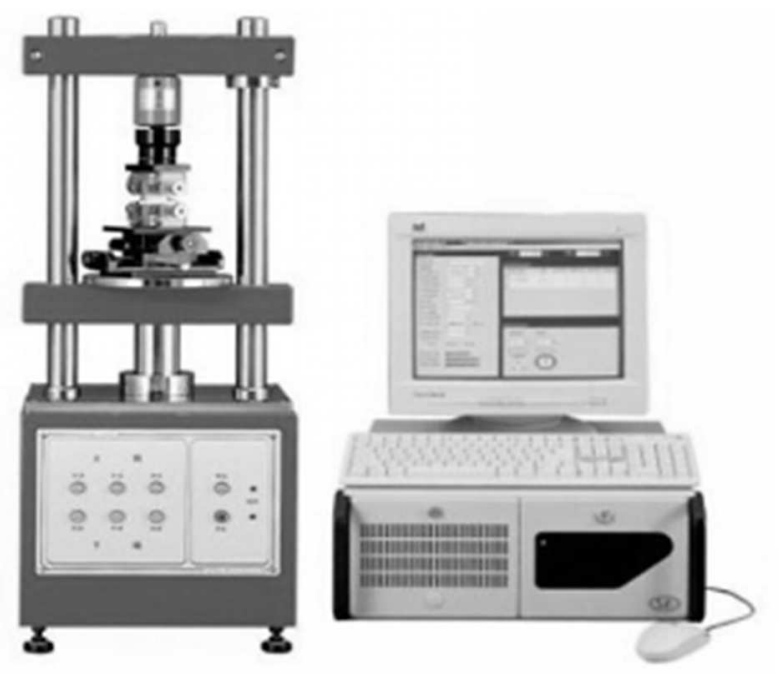

Fig. 4 Structure of the inserting force testing machine

Tab. 6 Test machine technical parameters

\begin{tabular}{ll}
\hline Minimum display stroke & $0.001[\mathrm{~mm}]$ \\
\hline Machine two-column inner & $200[\mathrm{~mm}]$ \\
distance & $5[\mathrm{kgf}]$ \\
Minimum display load & $0.2[\mathrm{gf}]$ \\
Load accuracy & $0.2 \%$ \\
Maximum test trip & $150[\mathrm{~mm}]$ \\
Test speed range & $1-200[\mathrm{~mm} / \mathrm{min}]$ \\
Transmission mechanism & Ball screw \\
Drive motor & Servo motor \\
External dimension & $360 * 260 * 940[\mathrm{~mm}]$ \\
Machine weight & $70[\mathrm{~kg}]$ \\
Using power & $\mathrm{AC} 110[\mathrm{~V}]$ or $220[\mathrm{~V}]$ \\
\hline
\end{tabular}


Fig. 5 can directly reflect that the peak value is 0.3114 , the contact reliability of fisheye contact is best when the value of pull force minus insert is maximum, the edge width is optimized to 0.74 [mm], the inner diameter width is optimized to 0.34 [mm]. The test results are close to the simulation results of response surface methodology, which verified the feasibility of this methodology.

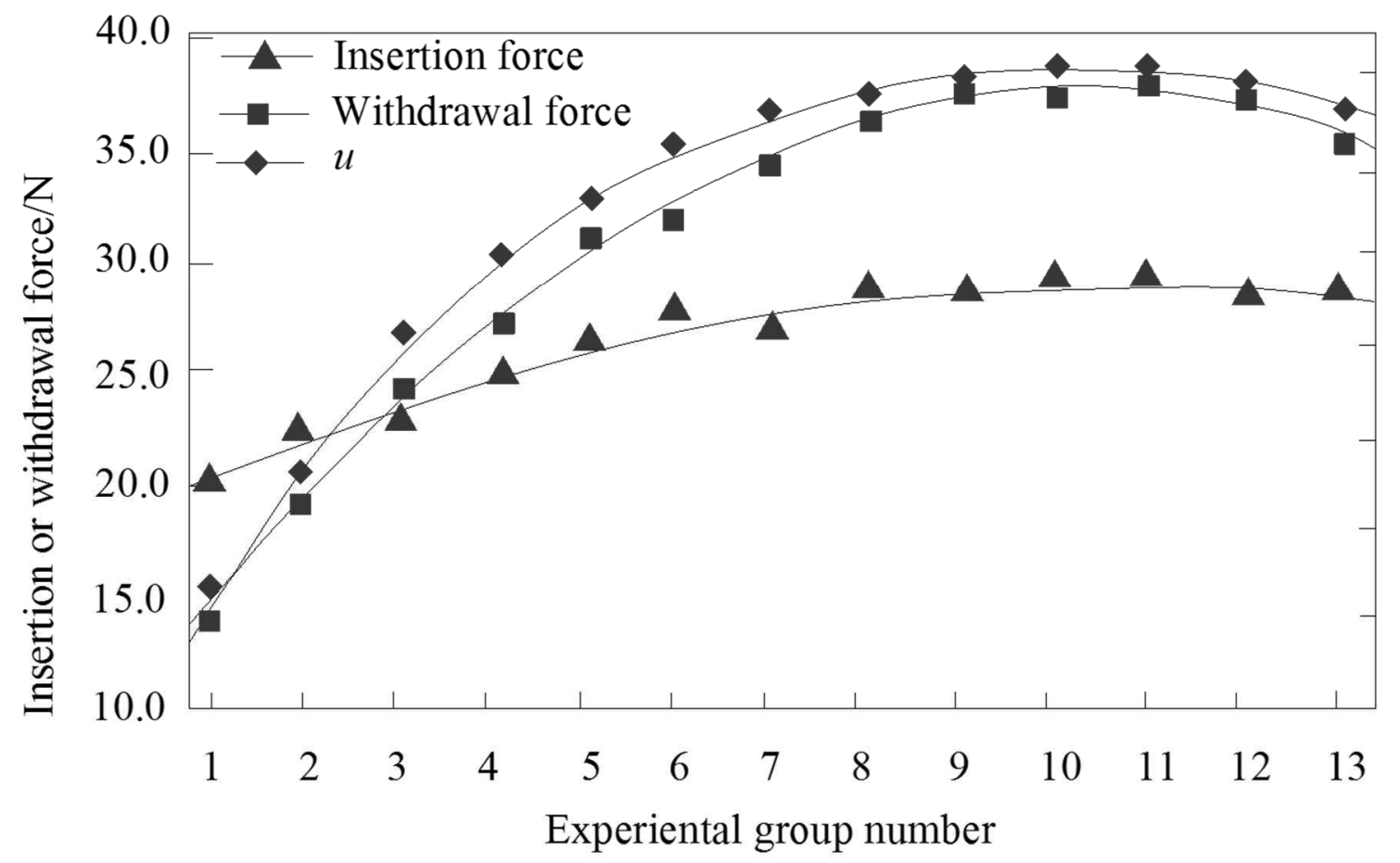

Fig. 5 Comparison of interpolation force, $u$ value simulation and test results

\section{Conclusion}

The response surface methodology is used to verify the reliability and accuracy of the fisheye contact structural optimization parameters, and the two factors modeling simulation shows that the response surface methodology can be applied to the optimization of other structural parameters.

The MATLAB is used to establish second-order response surface fitting test can obtain the data, which reflect fisheye structure parameters' effect on the withdrawal force accurately. Optimizing parameters by synthesizing fitting results, when we find outer rim width is 0.74 $[\mathrm{mm}]$, the inner diameter width is $0.32[\mathrm{~mm}]$, the structure is optimal. Moreover, we analysis the results practically through the experimental verification on this basis, which shows that the methodology is reliable, and can be applied in the field of structural analysis and so on, providing a theoretical methodology for optimal design.

\section{Acknowledgments}

The research is funded partially by Key Research and Development Program of Jiangsu Province (BE2018127) and Program for Student Innovation through Research and Training of Nanjing Agricultural University (1730A08).

\section{References}

[1] CHEN, W.H., KONG, X.Z. QIAN, P. GAO, L. ZHU, Z.P. (2016). Study on the effect of plug on the performance degradation of storage electrical connector. In: Mechanical and Electrical Engineering, Vol. 33, No. 5, pp. 507-512.

[2] YANG, F.W. (2012). Discussion on Innovation and Development of the Military Electrical Connector. In: Electromechanical Components, Vol. 2, No. 4, pp. 52-61.

[3] LING, S.Q., XU, L., FU, R., ZHAI. G.F. (2017). Optimization method of quality consistency of contact inserting force based on robust design principle. In: Chinese Journal of Mechanical Engineering, Vol. 53, No. 4, pp. 190-197.

[4] WANG, Y.K., MENGO, X.B.,(2014). Application of MATLAB in engineering graphics. In: Journal of Jilin Engineering Technology Normal College, Vol. 30, No. 10, pp. 59-64.

[5] LIN, R.J., CHEN, W.H., LIU, J. (2010). Feasibility study on accelerated performance degradation test of aerospace electrical connector. In: Engineering Design Journal, Vol. 17, No. 4, pp. 317-320.

[6] PAN, J., JIN, F.J., CHEN, W.H. (2013) Structural analysis and pluggable test of contact parts of electrical connector. In: China Mechanical Engineering, Vol. 24, No. 12, pp. 1197 - 1200.

[7] XU, X.M., JIANG, Y.P., LEE, H.P., CHEN, N. (2016). Sound Insulation Performance Optimization of Lightweight Sandwich Panels. In: Journal of Vibro engineering, Vol. 18, No. 4, pp. 
2574-258.

[8] REN, W.B., JIAO, Y.B. (2010). Electrical Connector Fretting wear Research. In: Electromechanical components, Vol. 30, No. 1, pp. 28-38.

[9] MARIA, B., MIROSLAV, B., JAN D., MILAN, S. (2018). Comparison of Analytical Stress Analysis and Numerical Calculation of Mobile Work Machine Part. In: Manufacturing Technology, Vol. 18, No. 2, pp. 190-193.

[10] LIANG, Y.Z., QIAO, Y.P., JIN, B., WU, Q., XU W.P. (2015). Research on the force of electrical contact between brush and brush. In: Journal of engineering design, Vol. 22, No. 3, pp. 290-294.

[11] PAN, J., JIN, F.J., CHEN, W.H., QIAN, P. (2013). Structural analysis and pluggable test of contact parts of electrical connector, In: China Mechanical Engineering, Vol. 24, No. 12, pp. 1636-1641.

[12] XU, X.M. JIANG, Y.P., LEE, H.P. (2017). Multiobjective optimal design of sandwich panels using a genetic algorithm. In: Engineering Optimization, Vol. 49, No. 10, pp. 1665-1684.

[13] ELOUFA, A. (2010). Numerical and experimental optimization of mechanical stress, contact temperature and electrical contact resistance of power automotive connector. In: International Journal of Mechanics, Vol. 4, No.4, pp. 94-104.

[14] CHEN, W.H., LIU, J., GAO, L. (2011). Accelerated Degradation Reliability Modeling and Test Data Statistical Analysis of Aerospace Electrical Connector. In: Chinese Journal of Mechanical Engineering, Vol. 24, No. 6, pp. 957-962.

[15] HUANG, B., LI, X.B., ZENG, Z., YANG, Y.Z. (2017). Numerical simulation and analysis of the pulling force of the contact parts of the high-speed back panel connector. In: Mechanical Design and Manufacture, Vol. 23, No.5, pp. 21-25.

[16] YORDI, K.B., SIGIT, Y.M., (2017) Optimization of Resistance Spot Welding Process using Response Surface Methodology and Simulated Anneali. In: Manufacturing Technology, Vol. 17, No. 4, pp. 434-440.

[17] LOU, S.M., WANG, Y.X. LU, S., SU, C.J., (2016). Extrusion Process Parameters Optimization for the Aluminum Profile Extrusion of an Upper Beam on the Train Based on Response Surface Methodology. In: Manufacturing Technology, Vol. 16, No. 3, pp. 551-557. 